\title{
A High Urinary Urobilinogen / Serum Total Bilirubin Ratio Reported in Abdominal Pain Patients Can Indicate Acute Hepatic Porphyria
}

\author{
Chengyuan Song \\ Shandong University Qilu Hospital \\ Shaowei Sang \\ Shandong University Qilu Hospital \\ Yuan Liu ( $\square$ liuyuan@qiluhospital.com ) \\ Shandong University Qilu Hospital https://orcid.org/0000-0003-4991-552X
}

Research

Keywords: acute hepatic porphyria, urinary urobilinogen, serum total bilirubin

Posted Date: June 14th, 2021

DOI: https://doi.org/10.21203/rs.3.rs-587707/v1

License: @) (i) This work is licensed under a Creative Commons Attribution 4.0 International License. Read Full License 


\section{Abstract}

Background: Due to its variable symptoms and nonspecific laboratory test results during routine examinations, acute hepatic porphyria (AHP) has always been a diagnostic dilemma for physicians. Misdiagnoses, missed diagnoses, and inappropriate treatments are very common. Correct diagnosis mainly depends on the detection of a high urinary porphobilinogen (PBG) level, which is not a routine test performed in the clinic and highly relies on the physician's awareness of AHP. Therefore, identifying a more convenient indicator for use during routine examinations is required to improve the diagnosis of AHP.

Results: In the present study, we retrospectively analyzed laboratory examinations in 12 AHP patients and 100 patients with abdominal pain of other causes as the control groups between 2015 and 2021. Compared with the control groups, AHP patients showed a significantly higher urinary urobilinogen level during the urinalysis $(P<0.05)$. However, we showed that the higher urobilinogen level was caused by a falsepositive result due to a higher level of urine PBG in the AHP patients. Hence, we used serum total bilirubin, an upstream substance of urinary urobilinogen synthesis, for calibration. A remarkable increase in the urinary urobilinogen/serum total bilirubin ratio was observed in AHP patients when compared to the control groups. The area under the ROC curve of this ratio for AHP was 1.000 (95\% confidence interval, $1.000-$ $1.000, P<0.01)$. A cutoff value of 3.22 for the urinary urobilinogen/serum total bilirubin ratio yielded a sensitivity of $100 \%$ and a specificity of $100 \%$ to distinguish AHP patients from the controls.

Conclusion: A reported high urinary urobilinogen level that was adjusted by the serum total bilirubin level (urinary urobilinogen/serum total bilirubin ratio) could be used as a sensitive and specific screening marker for AHP in patients with abdominal pain.

\section{Background}

Acute hepatic porphyria (AHP) is a rare but life-threatening disease. There are four classes of AHP: acute intermittent porphyria (AIP), hereditary coproporphyria (HCP), variegate porphyria (VP), and aminolevulinic acid dehydratase deficient porphyria (ADP) ${ }^{1}$. Since ADP is extremely rare and presenting a different laboratory results ${ }^{2}$, we mainly focused on the more common classes including AIP, HCP and VP in the present study.

Abdominal pain is the most common presentation during the attack of $\mathrm{AHP}^{3}$. However, due to the various symptoms and nonspecific routine laboratory test results, the diagnosis of AHP has always been a significant challenge for physicians, and a delay in diagnosis or even misdiagnosis is very common and causes severe outcomes in AHP patients ${ }^{3-5}$. According to a previous study, $58 \%$ of deaths happened in the first two acute attacks due to the delayed or even inappropriate treatments ${ }^{6}$. Meanwhile, recurrent attacks greatly increased the economic costs and generated anxiety in the patients. Suicide has also been reported in AHP patients ${ }^{5,6}$. Therefore, early diagnosis and prompt treatments may help reduce mortality in these patients.

At present, the examination of urinary porphobilinogen (PBG) is the key test in patients with suspected $\mathrm{AHP}^{7}$. As an unconventional examination, the urine PBG is detected only when the physicians realize the possibility of AHP. This pronounced dependency on the physician greatly compromises the diagnosis of this rare disease, especially in regional and community hospitals where a physician's experience is often limited. Therefore, it is of great importance to identify a more convenient indicator during routine examinations to improve the diagnosis of AHP.

In this retrospective study, we discovered for the first time that the urinary urobilinogen/serum total bilirubin ratio was greatly increased in the AHP group compared with the control groups, indicating its potential clinical value in diagnosing AHP. Furthermore, the sensitivity and specificity of this ratio were assessed to evaluate the performance of the urinary urobilinogen/serum total bilirubin ratio as a rapid screening marker of AHP.

\section{Methods}

\section{Study design and participants}

We performed a retrospective study and reviewed patients with AHP who were admitted to our hospital between 2015 and 2021. The study protocol was approved by the Clinical Research Ethics Committee of Qilu Hospital, Shandong University, China. Informed consent was obtained from the patient who donated the urine sample. No informed consent was required from the other eleven patients because we only retrospectively accessed a de-identified database for analysis purposes.

Patients were diagnosed as AHP if they met the following diagnostic criteria ${ }^{8}$,(i) acute attack symptoms: severe abdominal pain in the absence of significant abdominal tenderness and neuropathic symptoms such as seizures; and (ii) a positive result for urinary PBG or a 
genotype with a mutation in the hydroxymethylbilane synthase, protoporphyrinogen oxidase and coproporphyrinogen oxidase genes..

According to studies of AHP in China, the common misdiagnosis of AHP includes intestinal obstruction, pancreatitis, appendicitis, cholecystitis, and gallstones ${ }^{9}$. We randomly enrolled 100 patients with intestinal obstruction, pancreatitis, appendicitis, and gallbladder diseases as the control group. Patients with appendicitis $(\mathrm{N}=25)$ and gallbladder diseases $(\mathrm{N}=25)$ were surgically treated. Their classic symptoms and postoperative pathology results confirmed the diagnosis. The diagnosis of pancreatitis $(\mathrm{N}=25)$ was established by the presence of more than two of the following criteria: (i) abdominal pain consistent with pancreatitis; (ii) serum amylase and/or lipase greater than three times the upper limit of normal; and/or (iii) characteristic findings from abdominal imaging (e.g., exudation around the pancreas)

10. Intestinal obstruction ( $\mathrm{N}=25)$ was confirmed by clinical signs and symptoms and a triad of multiple air-fluid levels, distention of small bowel loops, and the absence of gas in the colon during the abdominal imaging plain upright abdominal radiography or $\mathrm{CT}$ scan ${ }^{11}$. Patients with a history of tumor, hemolytic disease, liver cirrhosis, splenomegaly, and autoimmune hemolytic anemia were excluded.

\section{Data Collection}

The medical history, physical examination, and blood and urinary biochemical results were collected from the Electronic Medical Records of the patients.

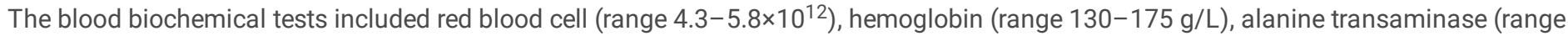
21-72 U/L), aspartate transaminase (range 17-59 U/L), albumin (range 35-50 g/L), total bilirubin (range 3-22 $\mu$ mol/l), conjugated bilirubin (range 0-5 $\mathrm{mmol} / \mathrm{l}$ ), unconjugated bilirubin (range 0-19 $\mu \mathrm{mol} / \mathrm{I}$ ), blood urea nitrogen (range 3.2-7.1 mmol/l), creatinine (range 58-133 $\mu \mathrm{mol} / \mathrm{I}$ ), lipase (range 3-300 U/L), amylase (range 30-110 U/L), serum potassium (range 3.6-5 mmol/l), serum sodium (range 137-145 $\mathrm{mmol} / \mathrm{l}$ ), serum chlorine (range 98-107 mmol/l), serum calcium (range 2.1-2.55 mmol/l), serum magnesium (range 0.7-1 mmol/l), and serum phosphorus (range $0.81-1.45 \mathrm{mmol} / \mathrm{I}$ ). These tests were performed using standard hospital laboratory techniques. BC-5390CRP (Mindray Biomedical Electronics Co., Ltd, China) was used for the RBC and hemoglobin examinations. The remaining serum markers were measured using a Vitros 5600 (Ortho Clinical Diagnostics, USA). Urinalysis was carried out using urine test strips and a Urilyzer Auto (Analyticon Biotechnologies AG, Germany).

\section{Statistical Analyses}

All the statistical analyses were performed in SPSS (version 24.0, SPSS, Chicago, IL, USA). Normally distributed continuous data (assessed by the Shapiro-Wilk test) are presented as mean \pm SD and were analyzed by the independent sample $t$-test. Non-normally distributed data are presented as median (percentage) and were analyzed by the Mann-Whitney U test. The diagnostic performance was measured as sensitivity, specificity, and accuracy. The cut-off for optimal clinical performance was determined by the receiver operator characteristic (ROC) curve. Results were considered significant at a $\mathrm{P}<0.05$.

\section{Results}

\section{Demographic and clinical characteristics of the AHP patients}

Between 2015 and 2021, 12 AHP patients were admitted into our hospital. The mean age at diagnosis was $27.73 \pm 6.77$ years old, with a female/male ratio of 11:1. These patients had $4.83 \pm 1.85$ acute attacks with a maximum of eight attacks before the correct diagnosis. Abdominal pain (100\%), pain in the extremities (50.00\%), seizures (33.33\%), and dark urine (16.67\%) were common clinical presentations. During the physical examinations, tachycardia and hypertension were observed in $75.00 \%$ and $58.33 \%$ of the patients, respectively. None of the patients reported skin lesions. In seven of the patients, AHP was triggered by hormonal variations during pregnancy or the menstrual cycle. In one patient, AHP was triggered by alcohol intake. No clear causes were recorded for the remaining four patients.

Interestingly, half of the AHP patients were first diagnosed by endocrinologist during a consult for hyponatremia. For the other six cases, four were diagnosed by neurologists, and two were diagnosed by a gastroenterologist and surgeon. During their previous visits, these patients had been misdiagnosed as intestinal obstruction (83.33\%), undifferentiated abdominal pain (75.00\%), appendicitis (8.33\%), and seizure (16.67\%). Appendectomy was performed in one patient at the primary hospital and seizures happened after the surgery.

Urinary urobilinogen was elevated in AHP patients, which was a false-positive result caused by urinary PBG

One hundred patients with abdominal pain caused by other diseases were enrolled in the control groups, and their laboratory results were compared with those of the AHP patients. During the acute attack, the AHP patients showed significantly lower hemoglobin, serum sodium, 
and serum chlorine, and higher urinary urobilinogen levels compared with control groups (Table 1). Urobilinogen is a product of bilirubin metabolism by anaerobic bacteria in the intestine. However, we found most of the AHP patients showed normal serum bilirubin levels in the present study (Table 1), which made it difficult to explain the elevated urinary urobilinogen values. Thus, we suspected that the elevated urinary urobilinogen level in AHP patients could have been a false-positive result caused by other substance that can react with Ehrlich's reagent on the dipstick. According to previous reports, sulfonamides, p-aminosalicylic acid, and drugs containing Azo dyes (nitrofurantoin, riboflavin, methyldopa) can also react with Ehrlich's reagent, but none of the patients in the current study had a history of taking these drugs.

Table 1

Comparison of laboratory results between acute hepatic porphyria patients and patients with abdominal pain due to other causes.

\begin{tabular}{|c|c|c|c|c|c|}
\hline & $\begin{array}{l}\text { Cholecystitis and } \\
\text { gallstones } \\
(\mathrm{N}=25)\end{array}$ & $\begin{array}{l}\text { Intestinal } \\
\text { obstruction } \\
(\mathrm{N}=25)\end{array}$ & $\begin{array}{l}\text { Pancreatitis } \\
(\mathrm{N}=25)\end{array}$ & $\begin{array}{l}\text { Appendicitis } \\
(\mathrm{N}=25)\end{array}$ & $\begin{array}{l}\text { Acute hepatic } \\
\text { porphyria } \\
(\mathrm{N}=12)\end{array}$ \\
\hline $\begin{array}{l}\text { Male/female } \\
\text { ratio }\end{array}$ & $13: 12$ & $10: 15$ & $13: 12$ & $10: 15$ & 1:11 \\
\hline Age & $67.00(60.50,69.00)^{\star \star}$ & $52.96 \pm 16.04^{\star \star}$ & $52.48 \pm 15.48 * \star$ & $42.80 \pm 17.97 * \star$ & $27.73 \pm 6.77$ \\
\hline RBC & $4.38 \pm 0.56$ & $4.40 \pm 0.60$ & $4.67 \pm 0.65^{\star \star}$ & $4.43 \pm 0.54^{\star}$ & $3.68 \pm 0.41$ \\
\hline Hemoglobin & $133.64 \pm 16.08^{\star}$ & $133.16 \pm 18.25^{\star}$ & $140.29 \pm 21.35^{\star \star}$ & $137.04 \pm 20.37 *$ & $107.75 . \pm 13.79$ \\
\hline ALT & $37.00(20.50,137.00)$ & $22.50(20.00,27.75)^{\star \star}$ & $63.50(33.50,233.50)$ & $\begin{array}{l}25.00(20.00 \\
42.00) \star \star\end{array}$ & $41.50(32.50,83.00)$ \\
\hline AST & $28.00(23.50,150.00)$ & $26.00(21.00,34.00)^{*}$ & $47.00(32.00,199.00)$ & $23.00(19.50,33.00)^{*}$ & $43.00(29.50,106.60)$ \\
\hline $\begin{array}{l}\text { Serum } \\
\text { albumin }\end{array}$ & $42.00(35.50,44.50)$ & $40.48 \pm 4.74$ & $44.00(36.50,46.00)$ & $41.36 \pm 6.28 *$ & $38.50 \pm 3.87$ \\
\hline Serum TBIL & $16.00(10.50,35.50)$ & $14.00(11.00,20.50)$ & $20.00(12.50,32.00)$ & $16.00(10.00,22.00)$ & $13.00(10.90,19.50)$ \\
\hline Serum DBIL & $0.00(0.00,0.00)$ & 0.00 & $0(0.00,0.00)$ & 0.00 & 0.00 \\
\hline Serum IBIL & $9.00(7.00,18.00)$ & $11.00(7.00,16.00)$ & $13.00(8.50,23.50)$ & $11.00(7.00,16.00)$ & $11.38 \pm 7.34$ \\
\hline $\begin{array}{l}\text { Serum } \\
\text { amylase }\end{array}$ & $69.50(57.25,83.00)$ & $72.00(59.00,72.00)$ & $531.00(238.00,1021.50)^{\star \star}$ & $75.50 \pm 34.00$ & $65.25 \pm 27.04$ \\
\hline $\begin{array}{l}\text { Serum } \\
\text { lipase }\end{array}$ & $66.00(37.25,162.00)$ & $31.00(23.00,56.00)$ & $2227.46 \pm 1649.60$ ** & $51.00(31.25,83.25)$ & $175.50 \pm 201.25$ \\
\hline $\begin{array}{l}\text { Serum } \\
\text { potassium }\end{array}$ & $4.00(3.70,4.29)$ & $3.86 \pm 0.66$ & $3.93 \pm 0.50$ & $3.97 \pm 0.44$ & $3.86 \pm 0.42$ \\
\hline $\begin{array}{l}\text { Serum } \\
\text { sodium }\end{array}$ & $136.43 \pm 3.37 * \star$ & $135.46 \pm 3.71 * \star$ & $136.25 \pm 3.08 * \star$ & $137.42 \pm 4.34^{\star \star}$ & $128.17 \pm 8.93$ \\
\hline $\begin{array}{l}\text { Serum } \\
\text { chlorine }\end{array}$ & $101.09 \pm 4.44^{\star \star}$ & $98.21 \pm 4.34^{\star \star}$ & $100.67 \pm 2.63^{\star \star}$ & 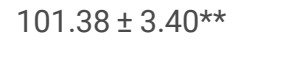 & $91.58 \pm 8.05$ \\
\hline $\begin{array}{l}\text { Serum } \\
\text { calcium }\end{array}$ & $2.29(2.07,2.33)$ & $2.27 \pm 0.16$ & $2.19 \pm 0.19$ & $2.27 \pm 0.19$ & $2.26 \pm 0.14$ \\
\hline $\begin{array}{l}\text { Serum } \\
\text { magnesium }\end{array}$ & $0.84 \pm 0.12^{\star}$ & $0.87(0.79,0.96) *$ & $0.82 \pm 0.09$ & $0.81 \pm 0.10$ & $0.69 \pm 0.22$ \\
\hline $\begin{array}{l}\text { Serum } \\
\text { phosphorus }\end{array}$ & $1.16 \pm 0.20$ & $1.31(1.26,1.54)$ & $1.13 \pm 0.35$ & $1.10 \pm 0.28$ & $1.24 \pm 0.32$ \\
\hline $\begin{array}{l}\text { Blood urea } \\
\text { nitrogen }\end{array}$ & $5.71 \pm 2.11$ & $5.81 \pm 2.17$ & $4.87 \pm 1.63$ & $4.64 \pm 1.32$ & $6.73 \pm 3.09$ \\
\hline $\begin{array}{l}\text { Serum } \\
\text { creatinine }\end{array}$ & $68.00(54.00,82.00))$ & $53.96 \pm 11.39$ & $59.17 \pm 21.96$ & $56.50(46.00,70.25)$ & $55.50(46.50,77.50)$ \\
\hline $\begin{array}{l}\text { Urinary } \\
\text { urobilinogen }\end{array}$ & $0.00(0.00,0.00)^{\star \star}$ & $0.00(0.00,0.00)^{\star \star}$ & $0.00(0.00,0.00)^{\star \star}$ & $0.00(0.00,0.00)^{\star \star}$ & $109.55 \pm 57.03$ \\
\hline \multicolumn{6}{|c|}{$\begin{array}{l}\text { ALT: alanine transaminase; AST: aspartate transaminase; DBIL: direct bilirubin; IBIL: indirect bilirubin; RBC: red blood cell;TBIL: total } \\
\text { bilirubin; }\end{array}$} \\
\hline & .01. & & & & \\
\hline
\end{tabular}


PBG, which is greatly increased during an acute attack of AIP, VP and HCP, can also react with Ehrlich's reagent. So we suspected that the elevated urinary urobilinogen level in AHP patients could have been a false-positive result caused by the urinary PBG and Watson-Schwartz test was used to confirm this speculation (Fig. 1). After an equal volume of Ehrlich's reagent was added, the urine sample of AHP patient turned red and proved the existence of PBG-Ehrlich compound or urobilinogen-Ehrlich complex (Fig. 1C). And then chloroform was added to the solution to distinguish these two substances. Red color in the aqueous phase of the test tube after adding chloroform illustrated the existence of the PBG-Ehrlich compound, whereas no color in the chloroform phase excluded the existence of the urobilinogen-Ehrlich complex (Fig. 1D).

\section{Performance of urinary urobilinogen/serum total bilirubin ratio as an indicator for AHP}

Because urinary urobilinogen was a false-positive result caused by urinary PBG, we used serum total bilirubin for calibration. A remarkable increase in urinary urobilinogen/serum total bilirubin ratio in AHP patients $(6.19 \pm 1.88$ vs. $0.00 \pm 0.0$, $P<0.01)$ was observed when compared with that in the control groups (Fig. 2). The performance of urinary urobilinogen/serum total bilirubin ratio as an indicator for AHP was assessed by generating a ROC curve (Fig. 3). Since one patient did not undergo the urinary examination, only data from eleven AHP patients were used for the ROC analysis. With the 100 samples from abdominal pain patients of other causes as the controls, the area under the ROC curve of urobilinogen/serum total bilirubin ratio for AHP was $1.000(95 \% \mathrm{Cl}, 1.000-1.000, \mathrm{P}<0.01)$. When using the maximum paired sensitivity and specificity values from the ROC curve, we determined a cutoff value of 3.22 for the urinary urobilinogen/serum total bilirubin ratio in detecting AHP from abdominal pain patients. The sensitivity, specificity, PPV, and NPV of the urinary urobilinogen/serum total bilirubin ratio to identify patients with AHP were $100 \%, 100 \%, 100 \%$, and $100 \%$, respectively.

\section{Discussion}

AHP is an autosomal dominant genetic disorder caused by the defect of the enzymes during heme biosynthesis pathway ${ }^{12}$. Due to the partial deficiency of these enzymes, excess amounts of 5-aminolevulinic acid (ALA) and PBG accumulate and cause dysfunction of the autonomic system and neuropathy ${ }^{13}$. Common presentations of AHP include abdominal pain (85-95\%), constipation (48-84\%), extremity pain (50$52 \%)$, nausea and vomiting (43-88\%), tachycardia (28-80\%), and hypertension (36-54\%) ${ }^{3}$. Bullous skin lesions may be present during an attack of VP or $\mathrm{HCP}^{14}$. In severe cases, seizures, hallucination and respiratory distress might also present and patients could require intensive care $^{15}$. In our study, $100 \%$ of patients complained of abdominal pain, which was much higher than that reported in previous studies. This might be due to a potential missed diagnosis of AHP patients with other symptoms caused by the poor awareness of physicians in our hospital.

The key issue in AHP management is to suspect the diagnosis ${ }^{16}$, and the elevation of urinary PBG is the main diagnostic criteria for AHP ${ }^{14}$. However, the urine PBG detection is only ordered and performed when AHP is suspected by physicians. In our study, we found that $50 \%$ of the patients were diagnosed by endocrinologist, which was rare in the published articles whose authors usually were from the neurology, general surgery or emergency departments. This finding highlights the importance of experienced physicians for AHP diagnosis. However, as a rare disease, awareness of AHP in China is limited ${ }^{8}$. According to a previous investigation carried out in a tertiary hospital in China, the misdiagnosis rate of AHP was 70\% in the hospital ${ }^{9}$. Further, case reports are barely reported from secondary or communal hospitals. Thus, a simple way for AHP screening is needed to help physicians notice the possibility of AHP.

Here, for the first time, we reported the important role of the urinary urobilinogen/serum total bilirubin ratio in the screening of AHP. Compared to the abdominal pain patients of other causes, the AHP patients showed a significantly higher urinary urobilinogen level and urinary urobilinogen/serum total bilirubin ratio. Urobilinogen is a product of bilirubin metabolism by anaerobic bacteria in the intestine. Up to $20 \%$ of the urobilinogen produced daily are reabsorbed from the intestine and undergo enterohepatic recirculation. The majority of the reabsorbed urobilinogen is taken up by the liver and then re-excreted into bile, while a small amount is excreted in the urine and being detected as urinary urobilinogen ${ }^{17}$. In healthy people, the urinary urobilinogen in urinalysis is negative since the amount of urobilinogen is too low to be detected. However, in certain diseases, such as hemolytic anemia, hepatic jaundice, and biliary disease, the serum bilirubin level is greatly elevated and leads to the excessive production of urobilinogen. Thus, a positive result shows in the urinalysis ${ }^{18,19}$. However, our study demonstrated that most of the AHP patients showed normal serum bilirubin levels, consistent with the literature $9,15,20-22$. Therefore, we believe that the elevated urinary urobilinogen level in AHP patients was a false-positive result caused by the urinary PBG, which was proved by Watson-Schwartz test.

The Watson-Schwartz test has been a widely used method for urinary PBG detection for more than 80 years. Ehrlich's reagent in the first step reacts with PBG and forms a red condensation product. However, PBG is not the only substance that can react with Ehrlich's reagent.

Urobilinogen, a substance being routinely examined in urinalysis, can also react with Ehrlich's reagent and form a red product ${ }^{23}$. As such, in the second step of Watson-Schwartz test, chloroform is added to the solution to distinguish the PBG-Ehrlich compound from the urobilinogen- 
Ehrlich complex. When chloroform is added, the red PBG-Ehrlich compound is located in the aqueous phase while the red urobilinogen-Ehrlich complex is located in the chloroform phase ${ }^{16}$.

At present, the dipstick method is the most widely used method of urinalysis. The Ehrlich's reagent pad on the dipsticks is used for detection of urinary urobilinogen. Following the manufacturer's instructions or using an automated dipstick reader, we can obtain semiquantitative results of urobilinogen. However, a false-positive result can occur due to urinary PBG since it is difficult to use chloroform to distinguish the red product on the dipstick. To avoid this, we used serum total bilirubin, which is a routine examinations and generally performed in patients with abdominal pain, for calibration. In the AHP patients in this study, the urinary urobilinogen increased greatly (caused by PBG) while the serum total bilirubin was normal or slightly elevated. This led to a great increase in the urinary urobilinogen/serum total bilirubin ratio. Via ROC curve analysis, we found that the cutoff point for AHP diagnosis was 3.22. So in patients with typical clinical symptoms such as abdominal pain, the AHP diagnosis should be considered when the urinary urobilinogen/serum total bilirubin ratio was above 3.22 and further investigation of urinary PBG and ALA should be carried out to confirm the diagnosis.

The potential clinical use of the urinary urobilinogen/serum total bilirubin ratio suggests the importance of urinalysis in the diagnosis of AHP. Dark urine is very common in AHP patients and sometimes becomes the first clue for AHP in the intensive care unit ${ }^{5,24}$. However, in the emergency department, dark urine color is often overlooked both by patients and physicians. In our study, all the urine specimens showed amber color during urinalysis, but only two patients mentioned a change of urine color in their complaints. In addition, a menstrual period is a common predisposing factor of an acute attack, but urinalysis is often avoided both by the physicians and female patients during their periods. Therefore, we strongly suggest that all patients with abdominal pain undergo urinalysis, and the urinary urobilinogen/serum total bilirubin ratio should be calculated for a diagnosis of potential AHP.

Our study has several limitations. Since AHP is a rare disease, we only enrolled 12 patients from one single center in this study. Due to the limited sample size, there might be variations in the cut-off point. In this retrospective study, although we enrolled patients whose diagnosis of diseases other than AHP were confirmed by postoperative pathological results, typical imaging and laboratory findings, no urinary PBG detection was done to fully rule out the possibility of AHP in the control groups. Future multi-center studies with larger sample sizes are needed.

\section{Conclusions}

In patients with abdominal pain, the reported high urinary urobilinogen level (from the Ehrlich's test) adjusted by the serum total bilirubin level (the urinary urobilinogen/serum total bilirubin ratio) can be used as an indicator for AHP. With a cutoff point of 3.22 , this ratio had a specificity and sensitivity of $100 \%$ and $100 \%$, respectively. When the ratio is higher than 3.22 , further investigation of urinary PBG, ALA or a genetic test is recommended. This finding may greatly improve the diagnosis of AHP, especially in primary or secondary hospitals where physicians have limited experience of this rare disease and the urine PBG detection is not available.

\section{Abbreviations}

AHP

acute hepatic porphyria; PBG:porphobilinogen; ALA:5-aminolevulinic acid; RBC:red blood cell; ALT:alanine transaminase; AST:aspartate transaminase; TBIL:total bilirubin; DBIL:direct bilirubin; IBIL:indirect bilirubin.

\section{Declarations}

Ethics approval and consent to participate

The study protocol was approved by the Clinical Research Ethics Committee of Qilu Hospital, Shandong University, China. Informed consent was obtained from the patient who donated the urine sample. No informed consent was required from the other eleven patients because we only retrospectively accessed a de-identified database for analysis purposes.

Consent for publication

Not applicable.

Availability of data and materials

The datasets used and analyzed in the present study are available from the corresponding author on reasonable request.

Competing interests

Page 6/10 
None.

Funding

None.

Authors' contributions

YL designed and conducted this study and was a major contributor in writing the manuscript. CS collected the data and drafted the manuscript. SS analyzed the data. All authors read and approved the final manuscript.

Acknowledgements

The authors wish to thank the physicians in the Department of Endocrinology, Department of Neurology, Department of Gastroenterology for diagnosing the AHP patients in this study.

\section{References}

1. Stolzel, U., Doss, M. O. \& Schuppan, D. Clinical Guide and Update on Porphyrias. Gastroenterology 157, 365-381 e364, doi:10.1053/j.gastro.2019.04.050 (2019).

2. Gouya, L. et al. EXPLORE: A Prospective, Multinational, Natural History Study of Patients with Acute Hepatic Porphyria with Recurrent Attacks. Hepatology 71, 1546-1558, doi:10.1002/hep.30936 (2020).

3. Cardenas, J. L. \& Guerrero, C. Acute intermittent porphyria: general aspects with focus on pain. Current medical research and opinion 34, 1309-1315, doi:10.1080/03007995.2018.1435521 (2018).

4. Liu, Y. P. et al. ED presentation of acute porphyria. The American journal of emergency medicine 23, 164-167, doi:10.1016/j.ajem.2004.03.013 (2005).

5. Bronisch, O., Stauch, T., Haverkamp, T., Beykirch, M. K. \& Petrides, P. E. Acute porphyrias: a German monocentric study of the biochemical, molecular genetic, and clinical data of 62 families. Annals of hematology 98, 2683-2691, doi:10.1007/s00277-019-03831-7 (2019).

6. Jeans, J. B. et al. Mortality in patients with acute intermittent porphyria requiring hospitalization: a United States case series. American journal of medical genetics 65, 269-273, doi:10.1002/(SICl)1096-8628(19961111)65:4<269::AID-AJMG4>3.0.C0;2-K (1996).

7. Deacon, A. C. \& Elder, G. H. ACP Best Practice No 165: front line tests for the investigation of suspected porphyria. Journal of clinical pathology 54, 500-507, doi:10.1136/jcp.54.7.500 (2001).

8. Yang, J. et al. Acute Intermittent Porphyria in the North of China: The Acute Attack Effect on Quality of Life and Psychological Condition. BioMed research international 2018, 3216802, doi:10.1155/2018/3216802 (2018).

9. Wang, Y., Chen, X. Y., Li, Y., Dong, X. H. \& Xu, F. [Clinical characteristics of 50 patients with acute intermittent porphyria]. Zhonghua nei ke za zhi [Chinese journal of internal medicine] 58, 520-524, doi:10.3760/cma.j.issn.0578-1426.2019.07.007 (2019).

10. Tenner, S., Baillie, J., DeWitt, J. \& Vege, S. S. American College of Gastroenterology guideline: management of acute pancreatitis. The American journal of gastroenterology 108, 1400-1415; 1416, doi:10.1038/ajg.2013.218 (2013).

11. Ten Broek, R. P. G. et al. Bologna guidelines for diagnosis and management of adhesive small bowel obstruction (ASBO): 2017 update of the evidence-based guidelines from the world society of emergency surgery ASBO working group. World journal of emergency surgery: WJES 13, 24, doi:10.1186/s13017-018-0185-2 (2018).

12. Anderson, K. E. Acute hepatic porphyrias: Current diagnosis \& management. Molecular genetics and metabolism 128, 219-227, doi:10.1016/j.ymgme.2019.07.002 (2019).

13. Duque-Serrano, L., Patarroyo-Rodriguez, L., Gotlib, D. \& Molano-Eslava, J. C. Psychiatric Aspects of Acute Porphyria: a Comprehensive Review. Current psychiatry reports 20, 5, doi:10.1007/s11920-018-0867-1 (2018).

14. Stein, P., Badminton, M., Barth, J., Rees, D. \& Stewart, M. F. Best practice guidelines on clinical management of acute attacks of porphyria and their complications. Annals of clinical biochemistry 50, 217-223, doi:10.1177/0004563212474555 (2013).

15. Sarala Kumari, D., Arumilli, M. N., Siva Kumar Reddy, L., Reddy, D. N. \& Motor, R. Acute Intermittent Porphyria Presenting with Posterior Reversible Encephalopathy Syndrome: A Rare Cause of Abdominal Pain and Seizures. Indian journal of critical care medicine: peerreviewed, official publication of Indian Society of Critical Care Medicine 24, 724-726, doi:10.5005/jp-journals-10071-23532 (2020).

16. Herrick, A. L. \& McColl, K. E. Acute intermittent porphyria. Best practice \& research. Clinical gastroenterology 19, 235-249, doi:10.1016/j.bpg.2004.10.006 (2005).

17. El-Guindi, M. A., El-Said, H. H., Hussein, M. H., Nassar Rel, S. \& Sira, A. M. Urinary urobilinogen in biliary atresia: A missed, simple and cheap diagnostic test. Hepatology research: the official journal of the Japan Society of Hepatology 46, 174-182, doi:10.1111/hepr.12558

Page $7 / 10$ 
(2016).

18. Bennink, R. J., Tulchinsky, M., de Graaf, W., Kadry, Z. \& van Gulik, T. M. Liver function testing with nuclear medicine techniques is coming of age. Seminars in nuclear medicine 42, 124-137, doi:10.1053/j.semnuclmed.2011.10.003 (2012).

19. Zgheib, H. et al. Utility of liver function tests in acute cholecystitis. Annals of hepato-biliary-pancreatic surgery 23, 219-227, doi:10.14701/ahbps.2019.23.3.219 (2019).

20. Bharti, S., Malhotra, P. \& Hirsch, B. Acute intermittent porphyria precipitated by atazanavir/ritonavir. International journal of STD \& AIDS 27, 1234-1235, doi:10.1177/0956462416633981 (2016)

21. Soundravally, R., Goswami, K., Nandeesha, H., Koner, B. C. \& Sethuraman, K. R. Acute intermittent porphyria: diagnosis per chance. Indian journal of pathology \& microbiology 51, 551-552, doi:10.4103/0377-4929.43759 (2008).

22. Cojocaru, I. M. et al. Acute intermittent porphyria-diagnostic and treatment traps. Romanian journal of internal medicine $=$ Revue roumaine de medecine interne 50, 33-41 (2012).

23. Watson, C. J., Bossenmaier, I. \& Cardinal, R. Acute intermittent porphyria. Urinary porphobilinogen and other Ehrlich reactors in diagnosis. JAMA: the journal of the American Medical Association 175, 1087-1091, doi:10.1001/jama.1961.63040120001010 (1961).

24. Patell, R., Dosi, R., Joshi, H., Shah, P. \& Tripathi, R. Challenges in the successful management of a case of acute intermittent porphyria in India. Tropical doctor 46, 150-153, doi:10.1177/0049475515610939 (2016).

\section{Figures}

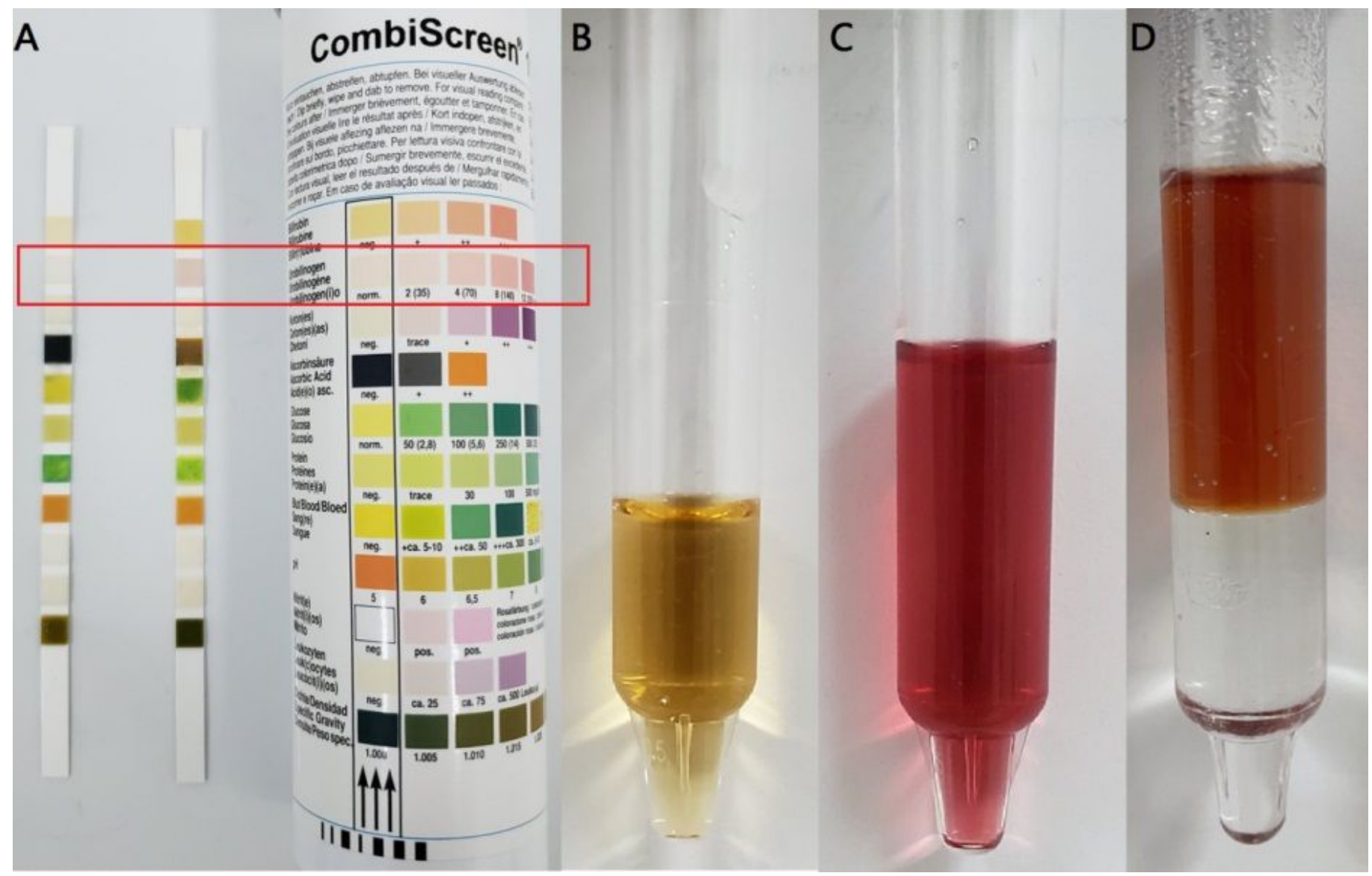

Figure 1

Elevated levels of urinary urobilinogen in acute hepatic porphyria patients were false-positive results caused by urinary PBG. (A) A positive urinary urobilinogen result reported by the dipstick (the right dipstick in the red box). (B) Fresh urine sample of AHP patient. (C) Urine sample turned red after adding an equal volume of Ehrlich's reagent. (D) Red color in the aqueous phase of the test tube after adding chloroform illustrated the existence of the PBG-Ehrlich compound, whereas no color in the chloroform phase excluded the existence of the urobilinogenEhrlich complex. 


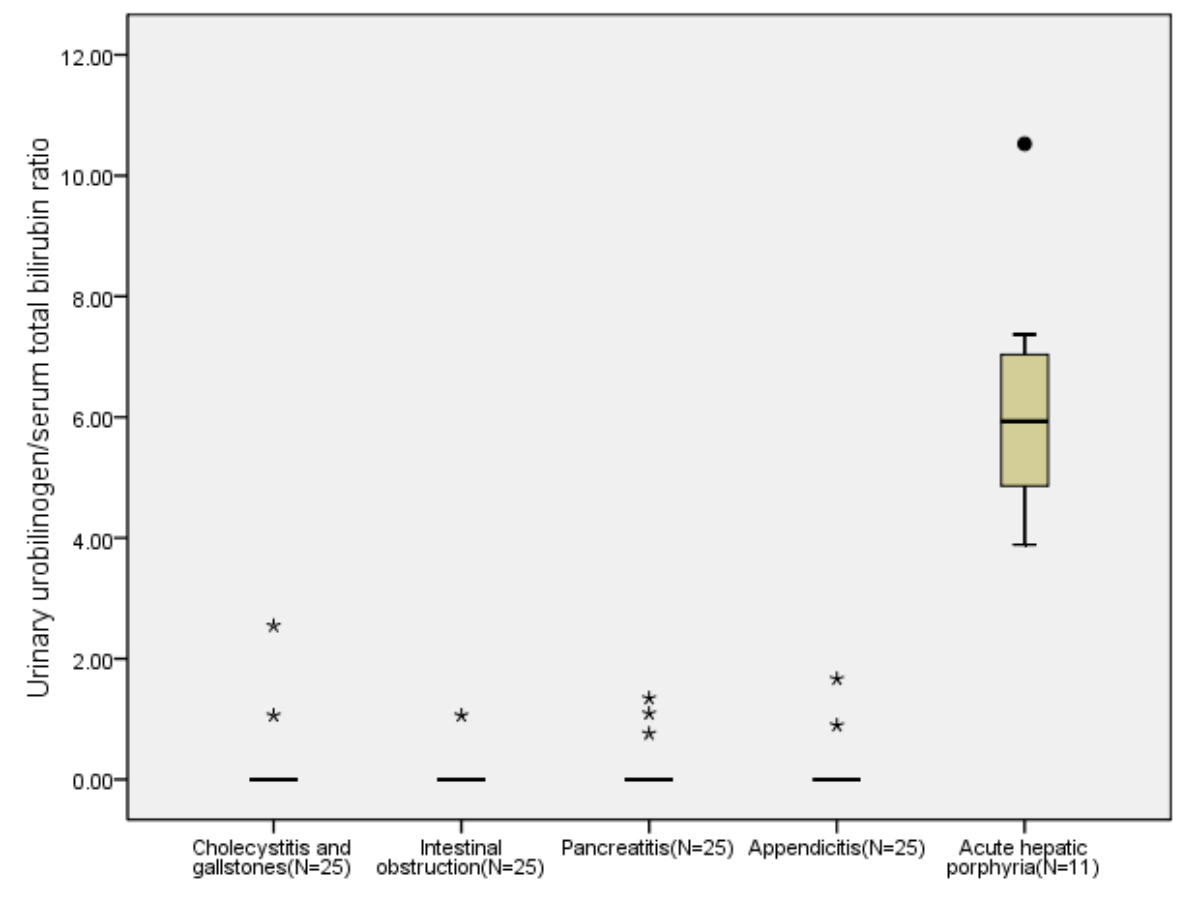

Figure 2

Comparison of urinary urobilinogen/serum total bilirubin ratio between acute hepatic porphyria patients and abdominal pain patients from other causes. A remarkable increase in urinary urobilinogen/serum total bilirubin ratio was observed in acute hepatic porphyria patients when compared with that in the control groups (6.19 \pm 1.88 vs. $0.00 \pm 0.0, P<0.01)$. Data are presented as mean $\pm S D$ median (percentage). An independent sample t-test was used for statistical comparisons.

ROC Curve

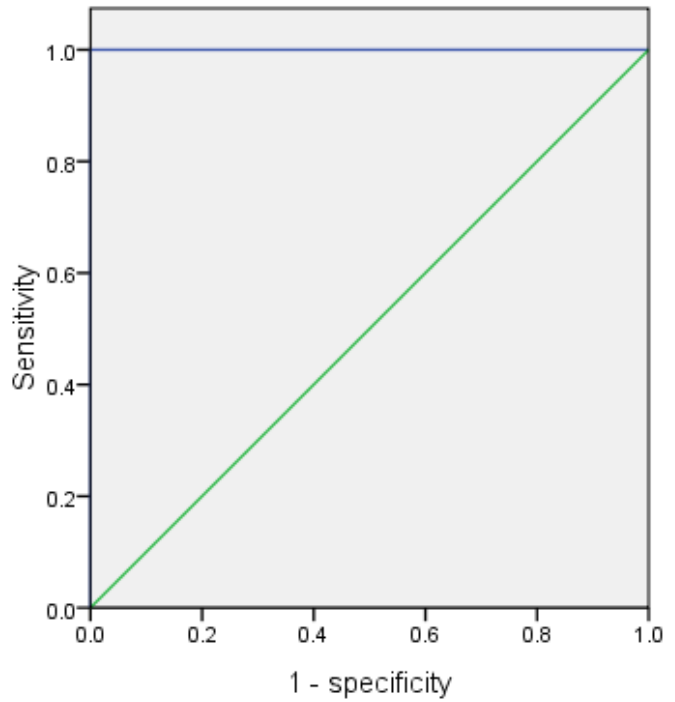

\section{Figure 3}

Diagnostic performance of urinary urobilinogen/serum total bilirubin ratio for acute hepatic porphyria. ROC curves were plotted using data from the acute hepatic porphyria patients and abdominal pain patients of other causes to assess the performance characteristics of urinary 
urobilinogen/serum total bilirubin ratio for acute hepatic porphyria. AUC was 1.000 with a $95 \% \mathrm{Cl}$ of $1.00-1.00$. Cutoff point $=3.22$

Page 10/10 